\title{
The future of mental health services research
}

\author{
GRAYSON NORQUIST
}

The field of services research has grown tremendously.in the last decade as the need for data about how to best organize, finance and deliver quality health services has grown. Although growth in the mental health area of services research started later than that in the general health area, it has increased substantially within the past five years. Data from mental health services research has been very influential for informing public policy on the delivery of mental health services in the United States.

\section{WHAT IS IT?}

Mental health services research is a multidisciplinary field and can be defined in a variety of ways. Although its boundary with clinical and other types of research is somewhat fluid, it has a targeted area of attention. It draws on methods and theory from various disciplines such as epidemiology, medicine, economics, sociology, operations research, psychology and biostatistics. The commonly used definition is: research that analyzes the impact of the organization, financing and management of health services on the cost, quality, access to and outcomes of health care. In addition, it is concerned with how well an intervention previously shown to work (i.e., has proven efficacy) performs in everyday practice settings with various types of clients (i.e., effectiveness of an intervention). These so-called effectiveness studies differ from efficacy studies that focus on whether an intervention (often unproven) works at all in a restricted population (e.g., clinical trials of new medications), because they are essentially a

Indirizzo per la corrispondenza: Dr. G. Norquist, NIMCH, Division of Epidemiology and Service Research, Room 10-105, 5600 Fisher Lane, Rockville, Maryland 20857 (USA).

Fax + 1-301-443.4045

E mail: gnorquis@nih.gov test of whether an efficacious intervention works under everyday conditions.

This description of services research is somewhat limited in that the areas addressed are broader than the definition implies. Topics range from economics and financing, families, research on mental health care in the legal system to primary care and clinical services research. Many projects also consider the effects of the cultural and social contexts on the delivery of services. A common misconception about services research is that it does not conduct randomized, controlled trials. The Rand Health Insurance Experiment is a prime example of such a trial in services research (Newhouse, 1974). Yet, it is true that many services research studies do not use randomized trials but instead find it necessary to address questions in populations that one either cannot or does not want to randomize.

\section{WHO FUNDS IT?}

A number of agencies within the US federal government support mental health services research. For example, the Health Care Financing Administrations (HCFA) provides some funds for studies that focus on specific financing issues and the Veterans Administration (VA) supports research related to its facilities. The newly created Center for Mental Health Services (CMHS) in the Substance Abuse and Mental Health Services Agency does not support services research but does provide funds for evaluation of innovative service demonstration projects. The Agency for Health Care Policy and Research (AHCPR) supports general health services research and in the past has funded some projects in mental health. However, due to recent budget cuts it has been able to fund only a few studies in the mental health area (primarily relating to mental health issues in the non-mental health setting). In addition, 
a number of private organizations (particularly insurance companies and managed care organizations) have set up their own services research organizations to focus on issues relevant to their particular organization. A few private foundations in the United States also fund specific projects in the mental health area. However, the vast majority of mental health services research in the United States is supported through grants from the National Institute of Mental Health (NIMH), a component of the National Institutes of Health (NIH).

In 1991, the NIMH National Advisory Mental Health Council issued a report, Caring for People with Severe Mental Disorders: A National Plan of Research to Improve Services (National Advisory Council, 1991) that called for a major expansion in mental health services research. Although this laid the ground work for what were important priorities in mental health services research, there were no new funds allocated to pursue most of the recommendations. In 1992, the US Congress, when it appropriated funds for NIMH research, requested that in fiscal year 1993 and following years, the NIMH spend $15 \%$ of its research budget on services research.

The NIMH has increased its funding of services research almost 2.5 times in the past decade. It spent approximately $6 \%$ of its budget in fiscal year 1986 on services research but increased the proportion to $15 \%$ of its budget (approx. $\$ 80$ million) by fiscal 1995 to comply with the Congressional directive. Corresponding to the increase in funds was an increase in the number and size of grants funded in the services research field. The NIMH funded approximately 30 grants in services research during fiscal year 1986 but by fiscal year 1995 it had increased that number by five times (150). The majority of these grants were for individual investigator grants but the NIMH also increased the number of services research centers and training grants it funded.

\section{WHAT HAS BEEN FUNDED?}

The portfolio of studies in mental health services research supported by NIMH during the past decade covers a wide range of topics. The NIMH National Advisory Council report mentioned above ( $\mathrm{Ca}$ ring for People with Severe Mental Disorders: $\mathrm{A} \mathrm{Na-}$ tional Plan of Research to Improve Services) targeted certain areas as priorities for research. One area of particular emphasis was research on improving mental health services for adults with severe and persistent mental disorders. In the United States there has been an effort for some years to ensure that people with such severe disorders receive coordinated mental health services in the community, especially as policy has shifted to move people to less restrictive environments from institutional inpatient settings. Research in this area has sought to develop a better understanding of how to organize and provide the best range of services. Some of the most promising research in this area has been the work on assertive community treatment. Research on these programs has shown them to be effective community interventions for people with the most severe disorders (Burns \& Santos, 1995). What has not been clear from research to date is what organizational systems work best to meet the needs of all people with mental disorders. Communities with varying political, social and cultural contexts need systems tailored to their populations and research must focus on how best to understand what services can meet those unique needs.

Another area that is important to improving services for those with severe mental disorders is research on rehabilitation. People with such disorders often have significant problems in social functioning. The goal of research in the rehabilitation area is to improve the capacity of individuals to function in their everyday world. Although a lot of work has been conducted in this area, more remains to be done before we will understand the best ways to set up rehabilitation services that improve the functional status of most people with severe disorders (Cook, 1995).

In the United States, many children do not receive mental health services and when they do they are usually in a variety of very different types of settings. The NIMH targeted children and adolescents as a major area of concern with the release of the $\mathrm{Na}$ tional Plan for Research on Child and Adolescent Mental Disorders in 1990 (National Advisory Council, 1990). This particular area of services research has grown the most in the past few years. Currently the NIMH has funded a number of grants and two services research centers that are conducting studies on the service needs of children and adolescents. In addition, the NIMH has recently funded a unique national study that will attempt to address a variety of questions about child and adolescent mental health services. This study known by the acronym UNOC-CAP (for use, need, outcomes and costs in 
child and adolescent populations) is a multi-site cooperative agreement between four academic institutions and the NIMH. The study will sample 10,000 children and adolescents between the ages of 4-17 in a national household probability sample. In addition, 3,200 children who are users of outpatient specialty and school based mental health services will be sampled, as will an extra sample of children and adolescents who are users of specialty inpatient and residential services. Both children and their care takers will be interviewed multiple times in a longitudinal design over two years. Interviews of the providers of services to these children and adolescents will also be conducted. Data from this study are expected to answer major services research questions for children and adolescents such as: What are their mental health needs? What barriers prevent access to services? How much do services for this population cost? What are the effects of various financing mechanisms on use of services? What are the outcomes of various treatment services? This study will be the largest national study with an epidemiological and mental health services research focus ever supported by the NIMH: Currently it is expected to start interviews in 1997.

Another area of major focus in mental health services research supported by the NIMH has been the area of economics. Most of the early economics research focused on estimates of the costs of mental disorders and the effects of various financing mechanisms on use of mental health services. Research in this area became a major focus with the advent of national health care reform proposals in the United States after the election of President Clinton. Early research was used in the formation of several plans considered during that time (National Advisory Mental Health Council, 1993; Arons et al., 1994). Even though the effort to reform health care on a national level has dwindled, the health care system in the United States has undergone significant changes in the past decade with the growth of managed care systems. The term managed care refers to a range of organizational, financial and regulatory arrangements that are used to control the utilization of health care services. The most common arrangements are capitated payments and restricted panels of providers with utilization review procedures. Most people in the United States now have health insurance that has some form of managed care. Some are covered under very restrictive arrangements that limit their access to specialty providers. The NIMH has recently encouraged research into mana- ged care and funded a research center to focus on this area.

The other area in services research that has received major support by the NIMH is research on mental health problems and care in the general health care sector (primary care). Early work in this area was focused on the lack of detection of mental disorders in the primary care setting. Recent work has moved to the development of methods to improve recognition of mental disorders in this setting and ways to improve treatment of mental disorders by primary care providers (Norquist \& Regier, 1996). This area has achieved special importance in the United States as many health plans now require their enrollees to first see a primary care provider before seeing a mental health specialist. For this and other reasons most people with mental health problems are seen in the primary care sector. Outside the United States, this has always been a major topic of interest and thus the United States has had a growing interface with other countries on research in this area. Recent work by the World Health Organization emphasized the importance of this research around the world (Ustün \& Sartorius, 1995).

The areas outlined above are those that have seen the greatest growth in the number of grants and have received the most funding during the past 10 years. In addition to these areas, there are a variety of other equally important topics but ones in which the number of grants has not been as large. The United States has a multi cultural population with a number of large ethnic and racial groups. Research on these populations has focused on understanding their mental health needs and ways to make mental health services more culturally appropriate. Those living in rural areas also have unique needs and the NIMH has funded research on ways to improve the delivery of services in those areas. Understanding the needs of families and ways to incorporate them into mental health services has also been an area of recent research. The NIMH is currently funding a number of grants that focus on distinguishing the mental health service needs of people who are infected with the AIDS virus. Researchers are also working to understand how to improve services for those who are homeless and those who are incarcerated in jails or prisons. Of increasing importance is research on how to improve services for those with co-occurring alcohol, substance abuse and mental disorders. Those with these co-occurring problems are often treated for their substance abuse problems in one sector and for their mental disorders in ano- 
ther. This has led to fragmentation of services and often inadequate treatments. Recent work has shown that it is possible to develop systems that can provide adequate services for this group of people (Teague et al., 1995).

\section{WHAT ARE FUTURE PRIORITIES?}

The NIMH will continue its strong commitment to services research and is likely to be the major source of funds for such research in the near future. Research on development of better systems of care for adults and children with severe mental disorders will continue to be a high priority. Economics and financing research will focus more on managed care as it will become a predominant part of the health care system in the United States. The other major area previously discussed that will continue to receive higher levels of funding is research on mental health problems in the general health care sector. Future research in this area will need to focus more on developing ways to improve recognition of significant mental health problems and most important, how to adequately treat such disorders in this setting.

There are several areas in mental health services research that to date have not had as much funding but are of crucial importance. The reason for lower funding is not because they were not priority areas but primarily because the number of researchers invested in these areas has not been as large as other areas mentioned above. The first of these is research on the aging population. In the United States the number of people over age 65 is expected to almost double in the early part of the next century. We need to have a better understanding of the needs of this population and the best ways to provide mental health services for them. The NIMH has asked for more grants relating to this population and will work to increase the number of researchers in this area.

Two other areas that up to now have received smaller amounts of funding but also will be of special importance in the years to come are: quality of care research and studies of the effectiveness of interventions (clinical services research). Quality of care research is concerned with understanding the factors that contribute to the differences in what is expected to be good clinical care and what actually happens
(McGlynn et al., 1988). This type of research has increased dramatically as many people have become concerned that under more restrictive systems of health care, clients are not receiving quality services. In the United States there has been an effort to develop measures of quality (e.g., outcomes measures) that can be used to judge how well providers are doing. In addition, researchers are working on the development of treatment guidelines, construction of better measures of quality of care, linking process to outcome through work on effectiveness of interventions, development of population based strategies to address the issue of access to care, improving measures of case mix and construction of better data sources. Some interesting results have come from these efforts such as finding differences in quality of care by setting and the demonstration of the cost-effectiveness of quality improvement (Norquist \& Wells, 1995; Sturm \& Wells, 1995).

Research on effectiveness of interventions is crucial if we are to implement the best possible services. Clinical trials with very selective populations have helped determine whether a specific intervention works at all (e.g., lithium for bipolar disorder). This type of research will remain very important but what we also need is to understand which interventions work best in various populations in everyday practice. People who participate in controlled clinical trials are not like those seen in everyday clinical practice who present with a variety of comorbid problems. Findings from such research allow us to apply the best possible interventions across the broadest number of people. Such studies can also help to influence clinical and basic research. If we know that certain types of people don't respond to a given medication then basic and clinical researchers can determine the unique characteristics of those populations and develop newer, more effective interventions for them.

Services research will need to pay particular attention to understanding the needs of consumers and their family members. A problem with past research in mental health has been the desire to focus on clinical symptomatology and diagnosis. Yet, diagnosis alone is not the major predictor of service needs. This realization has led to a greater emphasis on understanding all characteristics of the disablements associated with mental disorders. The NIMH has recently funded a joint project with the World Health Organization to develop ways of measuring the disablements of people with mental disorders. More work is needed to expand our knowledge about impairments, disabilities and handicaps in people with 
mental disorders and their impact on the use of mental health services.

Knowing what areas are important to fund seems obvious. The real challenge becomes how best to conduct studies to address these areas. Services research is a field that requires complicated sampling and statistical methods. The NIMH recently issued an announcement to call for research on new methods for conducting such complex studies. In addition, future studies will need to include larger samples across various settings. This will require collaborative projects among various research sites.

Mental health services research is a complex field but has a very specific goal to ensure that the best quality and most cost effective mental health services are delivered to those who need them so that people with these disorders have the best quality of life possible. No research study can address all aspects of this goal in a single study. Yet, the compilation of data from various studies can inform policy makers who must inevitably develop such systems of care. The NIMH expects that the portfolio of grants it funds will provide policy makers with the data they need to make such decisions in the future.

\section{REFERENCES}

Arons B.S., Frank R.G., Goldman H.H., McGuire T.G. \& Stephens S. (1994). Mental health and substance abuse coverage under health care reform. Health Affairs 13, 192-205.

Burns B.J. \& Santos A.B. (1995). Assertive community treatment:
An update of randomized trials. Psychiatric Services 46, 669675.

Cook J.A. (1995). Research on psychosocial rehabilitation services for persons with psychiatric disabilities. Psychotherapy \& $\mathbf{R e}$ habilitation Research Bulletin Summer, 5-11.

McGlynn E.A., Norquist G.S., Wells K.B., Sullivan G. \& Liberman R.P. (1988). Quality-of-care research in mental health: Responding to the challenge. Inquiry 25, 157-170.

National Advisory Mental Health Council (1990). National Plan for Research on Child and Adolescent Mental Disorders. Department of Health and Human Services Publication No. (ADM)90-1683. US Government Printing Office: Washington DC.

National Advisory Mental Health Council (1991). Caring for People with Severe Mental Disorders: A National Plan of Research to Improve Services. Department of Health and Human Services Publication No. (ADM)91-1762. US Government Printing Office: Washington DC.

National Advisory Mental Health Council (1993). Health care reform for Americans with severe mental illnesses: report of the National Advisory Mental Health Council. American Journal of Psychiatry 150, 1447-1465.

Newhouse J.P. (1974). A design for a Health Insurance Experiment. Inquiry 2, 5-27.

Norquist G.S. \& Regier D.A. (1996). The epidemiology of psychiatric disorders and the de facto mental health care system. Annual Review of Medicine 47, 473-479.

Norquist G.S. \& Wells K.B. (1995). Quality of care for depressed elderly patients hospitalized in the specialty psychiatric units or general medical wards. Archives of General Psychiatry 52, 695-701.

Sturm R. \& Wells K.B. (1995). How can care for depression become more cost-effective? Journal of American Medical Association 273, 51-58.

Teague G.B., Drake R.E. \& Ackerson T.H. (1995). Evaluating use of continuous treatment teams for persons with mental illness and substance abuse. Psychiatric Services 46, 689-695.

Ustün T.B. \& Sartorius N. (ed.) (1995). Mental Illness in General Health Care: An International Study. John Wiley \& Sons: Chichester. 